\title{
Quark mass effects on the topological susceptibility in QCD
}

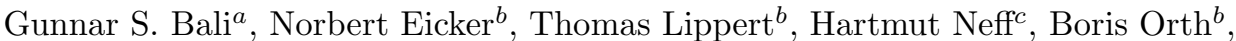 \\ Klaus Schilling ${ }^{b c}$, Thorsten Struckmann ${ }^{c}$ and Jochen Viehoff ${ }^{c}$ \\ ${ }^{a}$ Department of Physics 8 Astronomy, The University of Glasgow, Glasgow G12 8QQ, Scotland \\ ${ }^{b}$ Fachbereich Physik, Bergische Universität Wuppertal, D-42097 Wuppertal, Germany \\ ${ }^{c}$ von Neumann Institute for Computing, c/o Research Center Jülich, D-52425 Jülich and DESY, D-22603 Hamburg, Germany
}

(SESAM and $\mathrm{T} \chi \mathrm{L}$ Collaborations)

(October 29, 2018)

\begin{abstract}
We investigate topology in lattice simulations of QCD with two flavours of dynamical Wilson fermions. At various sea quark masses we find reasonable agreement between results for the topological charge from fermionic and gluonic definitions, the latter with cooling. We study correlations of the topological charge with light hadronic observables and find effects in the flavour singlet pseudoscalar channel. The quark mass dependence of the topological susceptibility, $\chi$, is consistent with the leading order small $m_{\pi}$ expectation, $\chi=f_{\pi}^{2} m_{\pi}^{2} / 4$.
\end{abstract}

PACS numbers: 11.15.Ha, 12.38.Gc, 11.30.Rd, 12.38.Aw

\section{INTRODUCTION}

One of the most intriguing features of QCD is its topological vacuum structure which results in phenomenological consequences with an important bearing on particle physics such as the breaking of the axial $U_{A}(1)$ and chiral symmetries.

In the context of pure gauge theories, lattice methods have by now matured to provide a valuable tool kit for accessing the continuum topological susceptibility, $\chi$. Various gluonic definitions of the topological charge lead to consistent estimates of $\chi$ [1], and remnants of the Atiyah-Singer index theorem are witnessed to hold on the lattice: the topological charge, $Q$, as obtained after cooling from the gluonic degrees of freedom is consistent with the fermionic index, as determined from counting the zero-level crossings of the eigenvalues of the Wilson-Dirac operator, $\gamma_{5}\left(\not D+m_{0}\right)$, under variations of the bare quark mass, $m_{0} \approx 0$ [2,3]. Moreover, one might perceive the very value of $\chi$ as delivered by quenched lattice simulations [4], $\chi_{q}^{1 / 4}=213(14) \mathrm{MeV}$, to confirm the lattice approach to topological properties: it turns out to be in fairly good agreement with the large $N_{c}$ anticipation made by Witten and Veneziano [8], $\chi_{q} \approx f_{\pi}^{2}\left(m_{\eta^{\prime}}^{2}+m_{\eta}^{2}-2 m_{K}^{2}\right) / 6 \approx(180 \mathrm{MeV})^{4}$. In conclusion, the lattice machinery appears to work for the study of topological aspects in gluodynamics.

Unfortunately, however, the situation regarding the
QCD vacuum proper is by far less settled: for sheer cost reasons so far only the regime of intermediate sea quark masses, not much lighter than the strange quark, has been explored. In fact it is still under debate whether the data from full QCD simulations support the expected vanishing susceptibility at chiral sea quark masses, $m \rightarrow 0$ :

$$
\chi=\frac{m}{n_{f}} \Sigma+\mathcal{O}\left(m^{2}\right), \quad \Sigma=-\lim _{m \rightarrow 0} \lim _{V \rightarrow \infty}\langle\bar{\psi} \psi\rangle .
$$

Attempts to verify this prediction via lattice simulations have a long history. While the first lattice studies in this direction with Kogut-Susskind (KS) sea quarks 912 could not reach conclusive results, later investigations did yield some qualitative evidence in favour of the expected decrease of $\chi(m)$ [13].

Quite recently, this problem has been revisited from three different sides - but the debate is still open: the CP-PACS [14 and UKQCD [15] collaborations employed improved Wilson fermionic and different gluonic actions while the Pisa group [16] operated with two and four flavours of KS fermions. A further data point for $n_{f}=2$ KS flavours has been obtained by Hasenfratz [17] (for a recent review see e.g. Ref. 18]). While both the Pisa group (working at an inverse lattice spacing $a^{-1} \approx 2 \mathrm{GeV}$ and bare quark masses $a m_{0} \geq 0.01$ ) and the CP-PACS collaboration (at $a^{-1} \approx 1.3 \mathrm{GeV}, m_{\pi} / m_{\rho}>0.59$ ) saw no evidence whatsoever in favour of the expected chiral behaviour, the UKQCD collaboration did verify (at $a^{-1} \approx 2 \mathrm{GeV}$ and $\left.m_{\pi} / m_{\rho}>0.57\right)$ a decrease of $\chi(m)$, consistent with theoretical expectations.

In this paper we shall present an analysis focused on this issue, based on the final statistics of our SESAM and $\mathrm{T} \chi \mathrm{L}$ samples of $\mathrm{QCD}$ vacuum configurations. The article is organised as follows. In Sect. II we describe the details of our simulation and methodology, including a comparison between fermionic and gluonic definitions of the topological charge. Preliminary results on this comparison, based on smaller statistical samples, have been reported by us previously [19]. In Sect. [II we investigate correlations between the topological charge and the hadron spectrum. Finally, in Sect. IV, we present the lattice data on the topological susceptibility. 
TABLE I. Simulation parameters. Unless an error is stated the autocorrelation times $\tau_{\text {int }}$ are lower estimates. The number of measurements is related to the number of trajectories by $n_{\text {meas }}=\nu_{\text {meas }} n_{\text {traj }}$, with $\nu_{\text {meas }}=1 / 10$ in the quenched case $(\beta=6.0), \nu_{\text {meas }}=1 / 24$ at $\kappa=0.1565, \nu_{\text {meas }}=1 / 2$ in the second series of measurements at $\kappa=0.1575, L_{\sigma}=16$ and $\nu_{\text {meas }}=1 / 25$ otherwise. The physical units have been obtained by setting $r_{0}^{-1}=394 \mathrm{MeV}$ and are subject to scale uncertainties of about $5 \%$. All unquenched simulations have been performed at $\beta=5.6$.

\begin{tabular}{cccccccc}
\hline \hline$\kappa$ & $L_{\sigma}$ & $n_{\text {meas }}$ & $r_{0} a^{-1}$ & $a_{\text {eff }}^{-1} / \mathrm{GeV}$ & $m_{\pi} a L_{\sigma}$ & $m_{\pi} / m_{\rho}$ & $\tau_{\text {int }}$ \\
\hline 0.1560 & 16 & 206 & $5.11(3)$ & $2.01(1)$ & $7.14(4)$ & $0.834(3)$ & 30 \\
0.1565 & 16 & 209 & $5.28(5)$ & $2.08(2)$ & $6.39(6)$ & $0.813(9)$ & 55 \\
0.1570 & 16 & 200 & $5.48(7)$ & $2.16(3)$ & $5.51(4)$ & $0.763(6)$ & 40 \\
0.1575 & 16 & 215 & $5.96(8)$ & $2.35(3)$ & $4.50(5)$ & $0.692(10)$ & 55 \\
$"$ & $"$ & 2200 & $"$ & $"$ & $"$ & $"$ & $54(4)$ \\
0.1575 & 24 & 150 & $5.89(3)$ & $2.32(1)$ & $6.65(6)$ & $0.704(5)$ & 140 \\
0.1580 & 24 & 140 & $6.23(6)$ & $2.45(2)$ & $4.77(7)$ & $0.574(13)$ & 130 \\
\hline$\kappa_{p h}$ & - & - & $6.73_{-19}^{+13}$ & $2.65_{-8}^{+5}$ & - & $0.179(1)$ & - \\
\hline$\beta=6.0$ & 16 & 320 & $5.33(3)$ & $2.10(1)$ & - & - & 35 \\
\hline \hline
\end{tabular}

\section{DETERMINATION OF THE TOPOLOGICAL CHARGE}

\section{A. Measurements}

We analyse ensembles of gauge configurations that have been generated by means of the hybrid Monte Carlo (HMC) algorithm using the Wilson fermionic and gluonic actions with $n_{f}=2$ mass degenerate quark flavours at the inverse lattice coupling, $\beta=5.6$, corresponding to an inverse lattice spacing $a^{-1}=\left(2.65_{-8}^{+5} \pm 0.14\right) \mathrm{GeV}$ at physical sea quark masses. This was done on $L_{\sigma}^{3} \times L_{\tau}=$ $16^{3} \times 32$ as well as on $24^{3} \times 40$ lattices at five different values of the sea quark mass parameter. The corresponding chiralities can be quantified in terms of $m_{\pi} / m_{\rho}$-ratios, ranging between $0.834(3)$ and $0.574(13)$ [20]. The relevant simulation settings are displayed in Table [1]. At each $\kappa$-value 4,000-5,000 thermalized HMC trajectories have been generated. In addition to the dynamical quark simulations, quenched reference measurements on $16^{4}$ lattices at $\beta=6.0$ were performed. The configurations that enter the analysis are separated by 25 (24 at $\kappa=0.1565$ and 10 in the quenched simulation) HMC trajectories.

Lower limits on the integrated autocorrelation times, $\tau_{\text {int }}$, are estimated by binning the data for the topological charge $Q_{i}, i=1, \cdots, n_{\text {meas }}$, into $n_{\text {bin }}$ blocks that contain $m$ successive measurements each. On each such block of length $m$ an average $\bar{Q}_{j}^{m}, j=1, \ldots, n_{\text {bin }}$, is calculated: $\bar{Q}_{j}^{m}=(1 / m) \sum_{i=1}^{m} Q_{m(j-1)+i}$. We determine fluctuations between these bins,

$$
\Delta Q_{m}^{2}=\frac{1}{n_{\mathrm{bin}}\left(n_{\mathrm{bin}}-1\right)} \sum_{j=1}^{n_{\mathrm{bin}}}\left(\bar{Q}_{j}^{m}-\langle Q\rangle\right)^{2}
$$

where the average $\langle Q\rangle \approx 0$ is calculated on the first $n_{\text {bin }} \times$ $m$ configurations. We then estimate the autocorrelation times,

$$
\tau_{\text {int }} \approx \frac{1}{2 \nu_{\text {meas }}} \max _{m^{2} \leq n_{\text {meas }} / 2}\left(\frac{\Delta Q_{m}^{2}}{\Delta Q_{1}^{2}}\right),
$$

that are included in the table. $\nu_{\text {meas }}$ denotes the measurement frequency. On the $16^{3} \times 32$ volume at $\kappa=0.1575$, in addition to the $\nu_{\text {meas }}=1 / 25$ time series, we also determined the topological charge with increased frequency $\nu_{\text {meas }}=1 / 2$, with a reduced number of 10 (as opposed to 60 ) cooling sweeps. The larger frequency enabled us to compute the autocorrelation time from the autocorrelation function itself, with full control over statistical errors [19]22]. The resulting value, $\tau_{\text {int }}=54(4)$, is consistent with our estimate obtained in the way described above, $\tau_{\text {int }} \approx 55$. We take this as an indication that our estimates are reasonable. Interestingly, the autocorrelation times grow both with decreasing quark mass and increasing volume as already observed in Ref. [19].

We employ a gluonic as well as a fermionic definition of the topological charge. In the gluonic case we define a topological charge density,

$$
q(x)=\frac{1}{16 \pi^{2}} \operatorname{tr} F_{\mu \nu}(x) \tilde{F}_{\mu \nu}(x)=\frac{1}{16 \pi^{2}} F(x) * F(x),
$$

where we use the symmetric (clover leaf) definition 23,

$$
F_{\mu \nu}=\frac{i}{4 a^{2}}\left(U_{\mu \nu}+U_{\nu,-\mu}+U_{-\mu,-\nu}+U_{-\nu, \mu}-4\right),
$$

and $\tilde{F}_{\mu \nu}=\frac{1}{2} \epsilon_{\mu \nu \rho \sigma} F_{\rho \sigma}$, which is correct up to order $a^{2}$ lattice artefacts. The topological charge,

$$
Q=\sum_{x} q(x)=\frac{1}{16 \pi^{2}}(F, * F),
$$

should then approach integer values as $a \rightarrow 0$ in the infinite volume limit, on sufficiently smooth gauge configurations.

\section{B. Renormalization}

In a quantum field theory, both $Q$ and $\chi$ undergo multiplicative renormalization. Moreover, $\chi$ requires additive renormalization. Cooling [24,25] is meant to filter for (semi)-classical features; indeed, after cooling, both renormalization constants have been shown to be close to their trivial values [1.5. In the context of this article we will not investigate properties of the density distribution $q(x)$ itself but rather stick to the net topological charge $Q$ only. For this purpose the iterative application of cooling by simple minimisation of the Wilson plaquette action is appropriate. To keep the cooling update local we visit the lattice sites in an even-odd pattern, rather than in the sequential ordering that is usually employed 
TABLE II. Estimates of the fermionic renormalization constant $Z_{P}$, Eq. (G), assuming the gluonic one to be unity.

\begin{tabular}{ccccc}
\hline \hline$\kappa$ & $L_{\sigma}$ & $m_{0} a$ & $Z_{P} m_{0} a$ & $Z_{P}$ \\
\hline 0.1560 & 16 & 0.0498 & 0.060 & 1.20 \\
0.1565 & 16 & 0.0395 & 0.056 & 1.43 \\
0.1570 & 16 & 0.0293 & 0.045 & 1.55 \\
0.1575 & 16 & 0.0192 & 0.029 & 1.49 \\
0.1575 & 24 & 0.0192 & 0.031 & 1.64 \\
0.1580 & 24 & 0.0092 & 0.017 & 1.79 \\
\hline \hline
\end{tabular}

in the Monte Carlo updating of pure gauge configurations. The inner-most loop within a cooling sweep runs across the directions $\mu$, and we carry out 60 such sweeps. In Fig. 1 w we illustrate the resulting numerical pattern of topological charges which cluster nicely around integer values as anticipated.

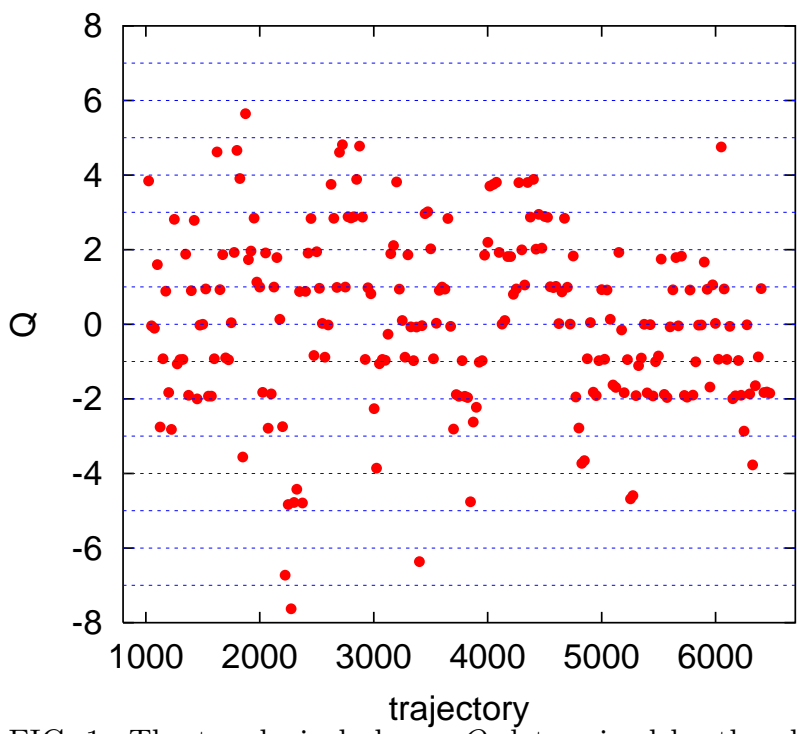

FIG. 1. The topological charge $Q$ determined by the gluonic definition, Eq. (6), after cooling on the $16^{3} \times 32$ lattices at $\kappa=0.1575$. The results are concentrated around integer values (dashed horizontal lines).

The fermionic method consists of determining the topological charge à la Smit and Vink [26],

$$
Q=\nu_{-}-\nu_{+}=-Z_{P} m_{0} \operatorname{tr} \gamma_{5} M^{-1},
$$

that is inspired by the continuum axial divergence relation, $\partial_{\mu}\left[\bar{\psi}(x) \gamma_{\mu} \gamma_{5} \psi(x)\right]=2 m \bar{\psi}(x) \gamma_{5} \psi(x)-2 n_{f} q(x)$. The above trace is to be taken over Dirac, colour and space-time indices only (not over the flavours), i.e. $\bar{\psi} \gamma_{5} \psi=n_{f} \operatorname{tr} \gamma_{5} M^{-1}$ for $n_{f}$ mass degenerate quark flavours. $\quad Z_{P}=\mathcal{O}(1)$ is a renormalization constant, $m_{0}=\left(\kappa^{-1}-\kappa_{c}^{-1}\right) /(2 a)$ denotes the bare quark mass and $M$ is the lattice discretised version of $\not D+m_{0}$,

$$
\begin{aligned}
2 \kappa M_{x y}=\delta_{x y} & -\kappa \sum_{\mu}\left[\left(1-\gamma_{\mu}\right) U_{x, \mu} \delta_{x+\hat{\mu}, y}\right. \\
& \left.+\left(1+\gamma_{\mu}\right) U_{x-\hat{\mu}, \mu}^{\dagger} \delta_{x-\hat{\mu}, y}\right]
\end{aligned}
$$

We determine $\operatorname{tr} \gamma_{5} M^{-1}$ using $Z_{2}$ noisy sources with diagonal improvement as detailed in Ref. 27. On the $\kappa=0.158$ configurations 100 such estimates were performed while at all other $\kappa$ values we averaged over 400 estimates

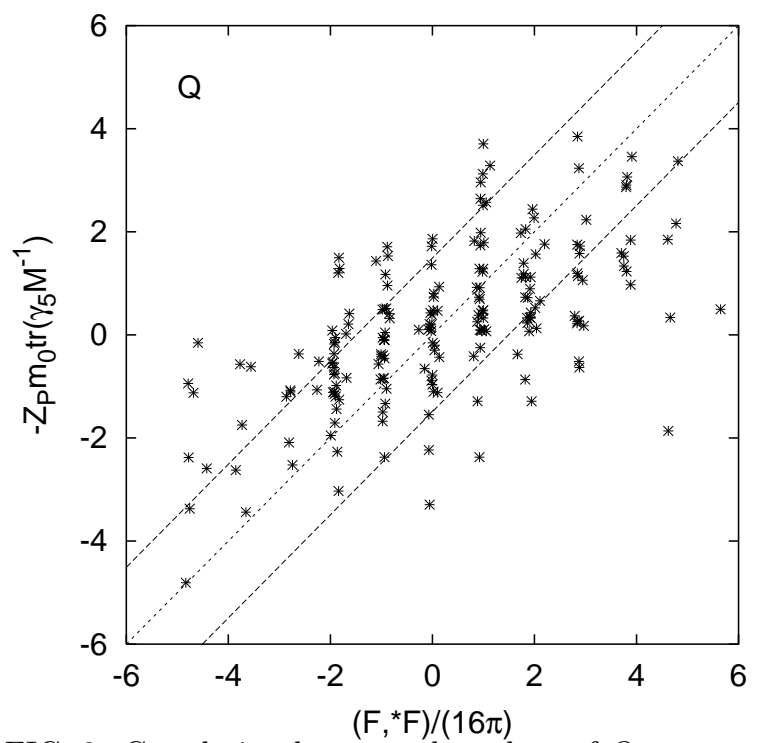

FIG. 2. Correlation between the values of $Q$ as computed from the gluonic [horizontal axis, Eq. (6)] and fermionic [vertical axis, Eq. (7), $Z_{P}=1.49$ ] definitions on $16^{3} \times 32$ lattices at $\kappa=0.1575$. The width of the error band corresponds to typical statistical uncertainties, due to the stochastic estimation of the fermionic values. $62 \%$ of the data points lie within this $1 \sigma$ region.

The renormalization constant $Z_{P}$ in Eq. (7) is unknown. We attempt to estimate the combination $Z_{P} m_{0} a$ from the ratio of the gluonic [Eq. (6)] and of the fermionic [Eq. (7) $]$ definitions. We determine $m_{0} a$ using the critical value, $\kappa_{c}=0.15849(2)$ [20]. The results are displayed in Table II, assuming the multiplicative renormalization of the gluonic definition after cooling to be unity. The estimates of the fermionic traces are subject to statistical uncertainties, $\Delta Q / Z_{P} \approx 1$ on the $16^{3} \times 32$ lattices and $\Delta Q / Z_{P} \approx 2-3$ on the $24^{3} \times 40$ volumes. In addition, both definitions are expected to suffer from different $\mathcal{O}(a)$ lattice artefacts. We do not attempt to estimate the resulting statistical and systematic uncertainties on $Z_{P}$. We find $Z_{P}$ to be of order one and to depend monotonously on the quark mass, with the deviation from unity increasing when approaching the chiral limit.

The correlation between the two definitions is visu-

\footnotetext{
${ }^{1}$ Approximating $Q$ by a finite number of noise vectors can result in an underestimated integrated autocorrelation time. Nonetheless, we find the $\tau_{\text {int }}$-estimates from the fermionic definition 22 to be consistent with the gluonic ones of Table If
} 
alised in the scatter plot, Fig. 2, for the $16^{3} \times 32$ lattices at $\kappa=0.1575$. While the gluonic data points cluster around integer values, this is not the case for the (stochastically estimated) fermionic values. The data of the figure are normalised such that the points should collapse onto the line with slope one. Indeed, 137 out of 199 values (62\%) lie within the one $\sigma$ error band. The correlation between the two definitions is also visible from the Monte Carlo histories depicted in Fig. 3 .

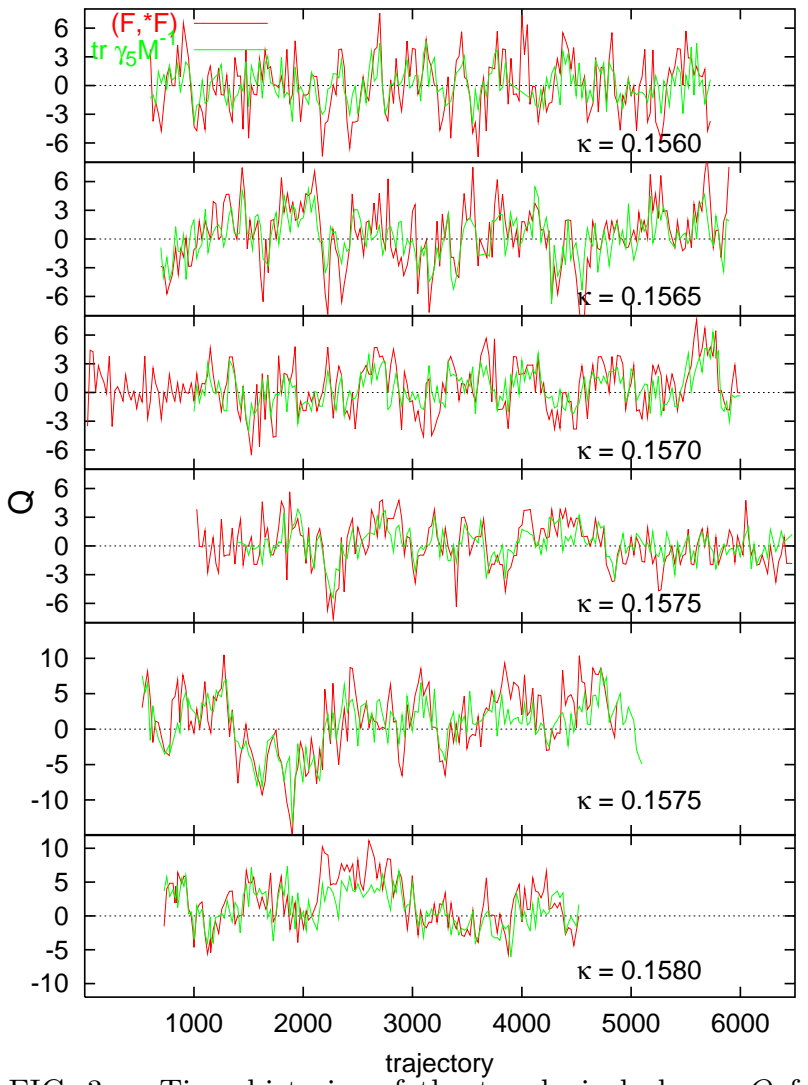

FIG. 3. Time histories of the topological charge $Q$ for the gluonic $[(F, * F)$, Eq. (6) $]$ and the fermionic $\left[\operatorname{tr} \gamma_{5} M^{-1}\right.$, Eq. (7)] definitions. The last two series have been obtained on $24^{3} \times 40$ lattices, the first four on $16^{3} \times 32$ volumes.

\section{Ergodicity}

In Fig. 3 we survey the Monte Carlo histories of $Q$ for all our runs. These charts provide a gross evidence for the quality of our data in view of the decorrelation with respect to the topological sectors. The topological susceptibility,

$$
\chi=\sum_{x}\langle q(x) q(0)\rangle=\frac{\left\langle Q^{2}\right\rangle}{V},
$$

should be independent of the volume $V=L_{\sigma}^{3} \times L_{\tau} a^{4}$ to a first approximation. Therefore, the modulus of the topological charge $\langle|Q|\rangle$ should scale in proportion to $\sqrt{V}$. In- deed, the topological charge distribution on the large lattice at $\kappa=0.1575$ is by a factor of about two wider than that on the small lattice. We also observe reduced fluctuations as we increase $\kappa$ at fixed $\beta$. While the $16^{3} \times 32$ time histories appear to tunnel ergodically through all topological sectors the total number of tunnellings observed for $\kappa=0.1580$ is not yet sufficient to achieve a symmetric distribution.

\section{TOPOLOGY AND THE HADRON SPECTRUM}

We address the question whether the statistics presented in Fig. 3 suffices to expose a significant $|Q|$ dependency of hadronic states. To minimise statistical errors we subdivided each sample into two subsamples only, one containing configurations with $|Q| \leq 1.5$ and one with $|Q|>1.5$. For the smaller volume at $\kappa=0.1575$ this division results in two subsamples of approximately 100 configurations each.

The most interesting test case is given by the effective masses in the flavour singlet pseudoscalar channel, $m_{\eta^{\prime}}^{\text {eff }}(t)$, that should be particularly sensitive to the vacuum topology. This is confirmed by the data displayed in Fig. 1 . We find the $\eta^{\prime}$ mass on vacuum configurations with $|Q| \leq 1.5$ to systematically lie below the corresponding mass obtained with the cut $|Q|>1.5$. Note that in the infinite volume limit one would expect such a sensitivity of correlation functions on $|Q|$ to disappear. For further details we refer to Ref. [28].

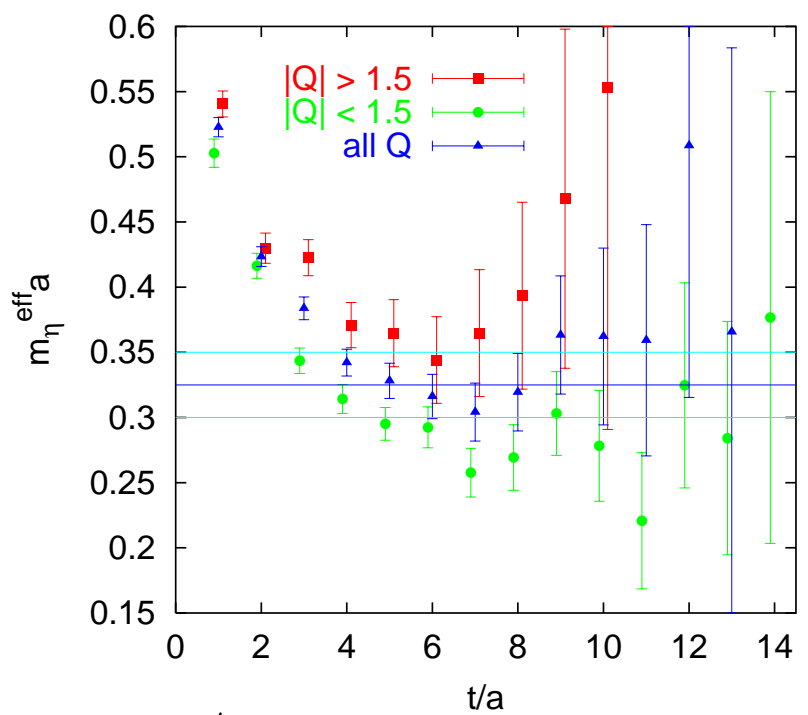

FIG. 4. The $\eta^{\prime}$ effective mass from a local-smeared correlation function on the $16^{3} \times 32$ lattice at $\kappa=0.1575$, separately determined on configurations with topological charge $|Q| \leq 1.5$ and $|Q|>1.5$. The horizontal error band is the fitted asymptotic mass obtained on the full sample [28].

In Fig. 5 we show the corresponding flavour octet channel quantities, i.e. $\pi$ effective masses, where we anticipate 
no such correlation with topology. This is indeed borne out by the data: not only at $t \rightarrow \infty$ but time slice by time slice no sensitivity to the value of the modulus of the topological charge is detected, with rather high statistical accuracy. The horizontal line with error band indicates the asymptotic large $t$ results obtained from a fit to the entire data sample [20].

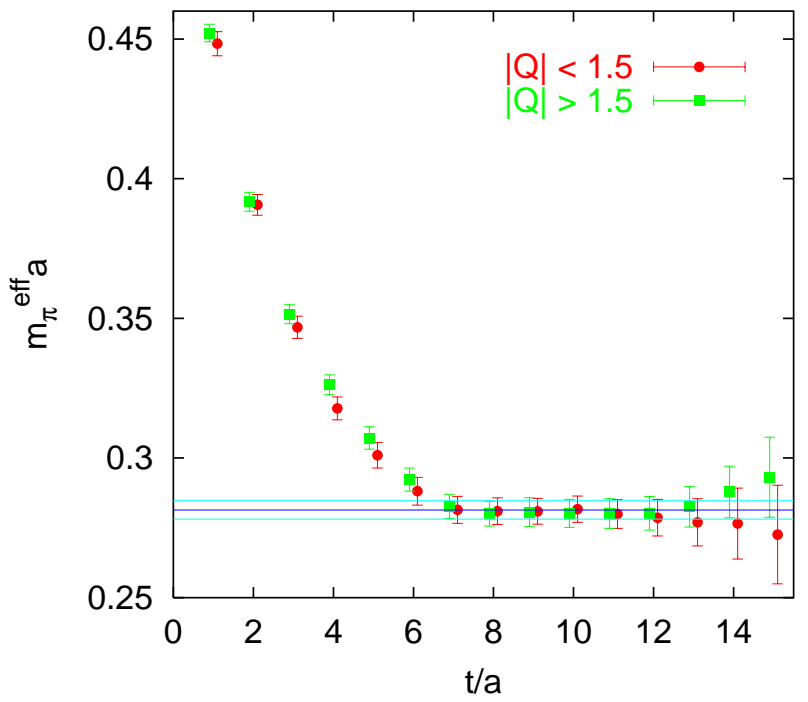

FIG. 5. The same as Fig. 4 for the $\pi$ mass.

Apart from the $\eta^{\prime}$ none of the standard mesonic, baryonic and glueball-like states exhibit correlations between $|Q|$ and the respective effective masses. In Figs. 6-8 we illustrate this for the $\rho$, the nucleon and the $\Delta$. In no case have we found any systematic effects on the static potential. Of course this does not exclude the possibility of correlations between hadronic properties and the distribution of instantons and anti-instantons and their relative orientations in colour and position space [29].

\section{THE TOPOLOGICAL SUSCEPTIBILITY}

In Figs. 9 - 11 we display three histograms of topological charge distributions. Each bin with width $\delta Q$, centred around $Q_{j}=j \delta Q$, contains all measurements resulting in charges within the interval $\left[Q_{j}-\delta Q / 2, Q_{j}+\delta Q / 2\right]$, where $\delta Q=1$ on the $16^{3} \times 32$ lattice depicted in Fig. 9 and $\delta Q=3$ for the $24^{3} \times 40$ lattices of Fig. 10 and Fig. 11. In addition to the data we display Gaussian distributions,

$$
n(Q)=\frac{n_{\text {meas }} \delta Q}{\sqrt{2 \pi\left\langle Q^{2}\right\rangle}} \exp \left(-\frac{Q^{2}}{2\left\langle Q^{2}\right\rangle}\right) .
$$

In Fig. 9 we include the statistical uncertainties of the individual bins, $\Delta n=2 \tau_{\text {int }} \nu_{\text {meas }} \sqrt{n}$, while in Fig. 10 and Fig. 11 the error on the width of the distribution is reflected by the error band around the central curve.

The fitted topological susceptibilities $\chi=\left\langle Q^{2}\right\rangle / V$ as well as $\langle Q\rangle$ are displayed in Table [II]. For orientation,

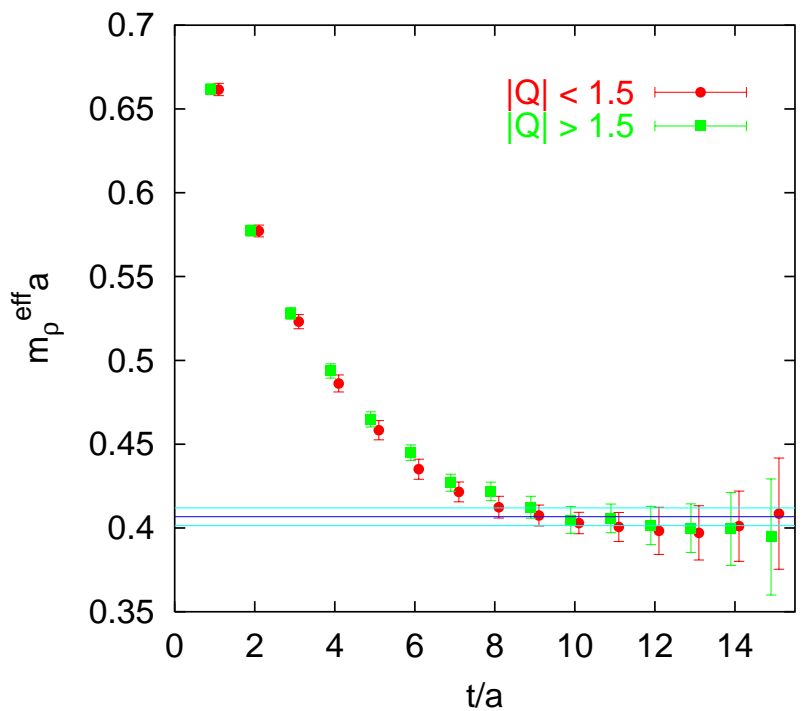

FIG. 6. The same as Fig. 1 for the $\rho$ mass.

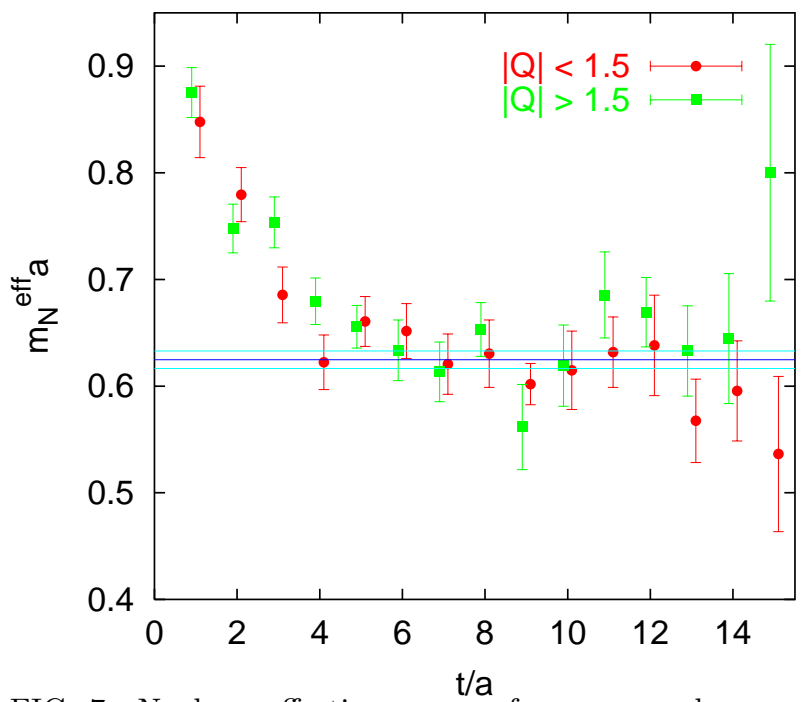

FIG. 7. Nucleon effective masses from smeared-smeared correlation functions.

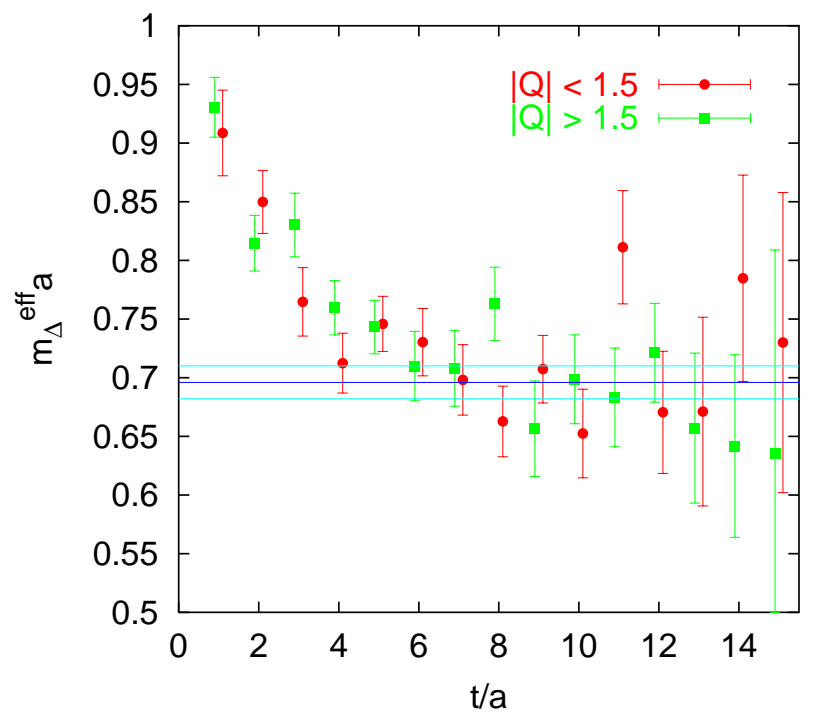

FIG. 8. The same as Fig. 0 for the $\Delta$ mass. 
TABLE III. Topological susceptibilities.

\begin{tabular}{cccccc}
\hline \hline$\kappa$ & $L_{\sigma}$ & $\langle Q\rangle$ & $\chi a^{4} / 10^{-6}$ & $\chi^{1 / 4} r_{0}$ & $\chi^{1 / 4} / \mathrm{MeV}$ \\
\hline 0.1560 & 16 & $0.04(32)$ & $69(6)$ & $0.466(11)$ & $183(4)(9)$ \\
0.1565 & 16 & $0.48(48)$ & $83(13)$ & $0.505(22)$ & $199(8)(10)$ \\
0.1570 & 16 & $0.60(34)$ & $57(10)$ & $0.476(22)$ & $187(8)(9)$ \\
0.1575 & 16 & $0.05(33)$ & $41(7)$ & $0.472(21)$ & $186(8)(9)$ \\
0.1575 & 24 & $0.84(1.33)$ & $44(7)$ & $0.479(20)$ & $189(8)(10)$ \\
0.1580 & 24 & $1.92(1.01)$ & $31(12)$ & $0.465(44)$ & $183(17)(9)$ \\
\hline quenched & 16 & $-0.68(33)$ & $80(14)$ & $0.505(22)$ & $199(9)(10)$ \\
\hline \hline
\end{tabular}

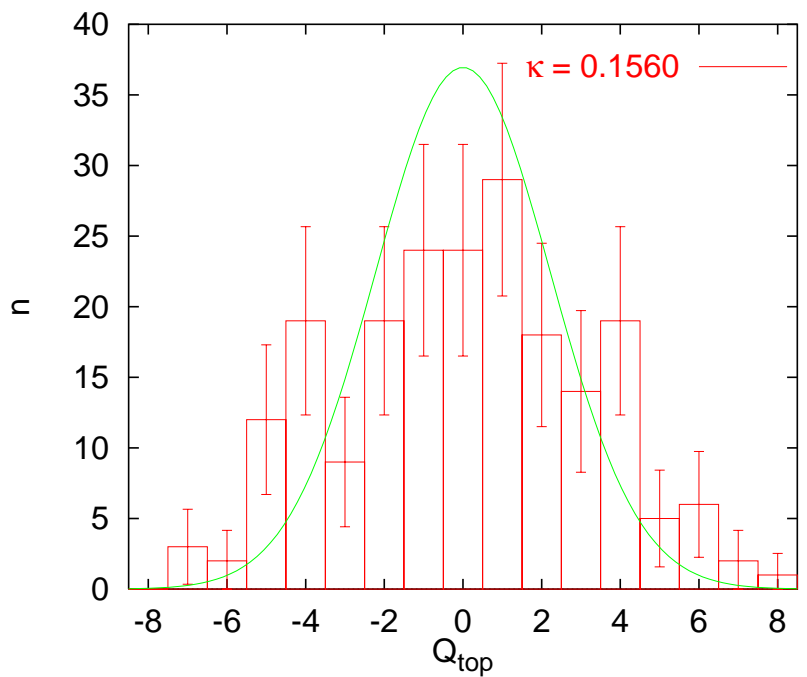

FIG. 9. Topological charge distribution at $\kappa=0.156$.

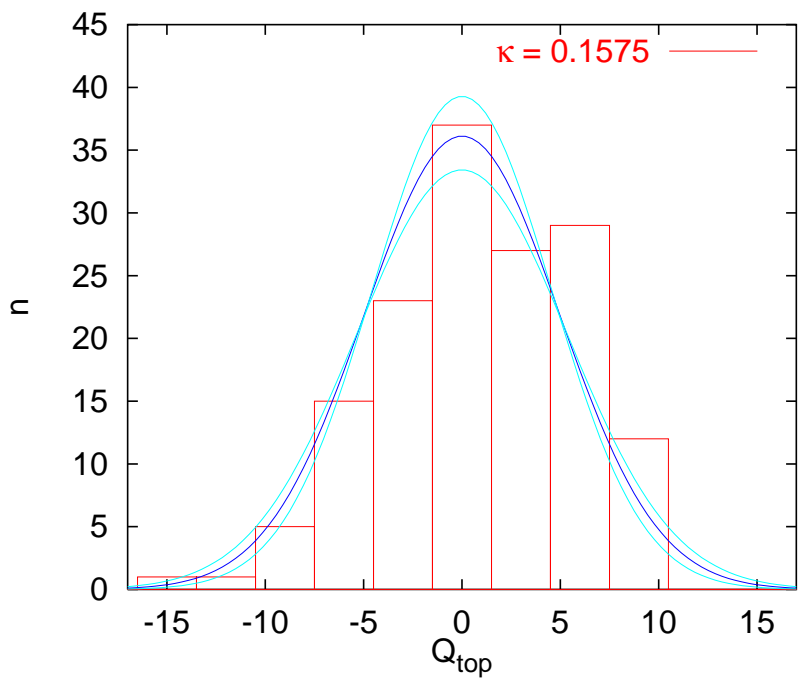

FIG. 10. The same as Fig. 9 for $\kappa=0.1575, L_{\sigma}=24$.

we convert $\chi$ into physical units in the last column of the table, using $r_{0}^{-1}=(394 \pm 20) \mathrm{MeV}$. The distribution at $\kappa=0.158$ is not symmetric around zero anymore, as reflected by the value $\langle Q\rangle=1.9 \pm 1.0$ and by Fig. 11 . Therefore, in this case, the resulting value of $\chi$ should pass with a grain of doubt, notwithstanding the comfortable error bars. A comparison between the $L_{\sigma}=16$ and $L_{\sigma}=24$ results at $\kappa=0.1575$ reveals that the level of

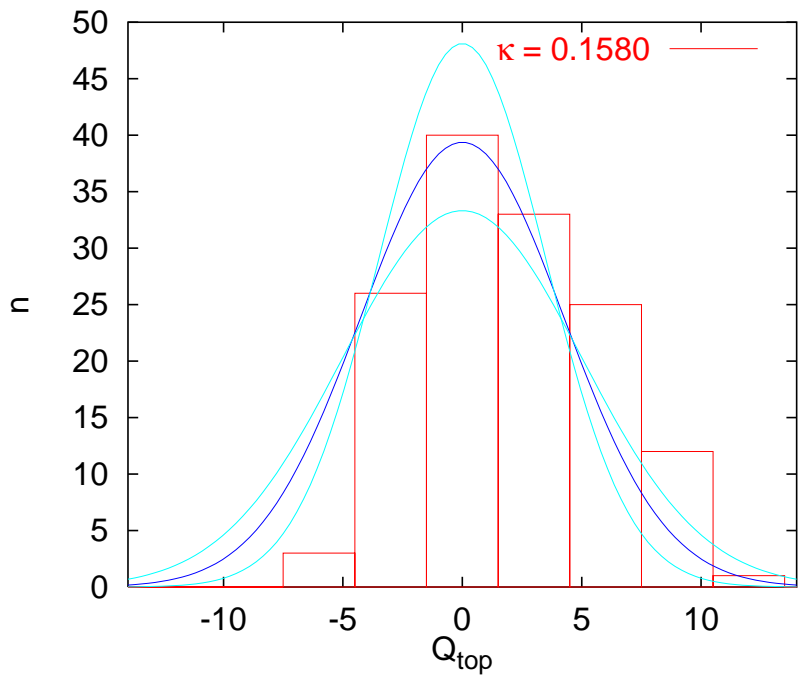

FIG. 11. Topological charge distribution at $\kappa=0.158$.

finite size effects on $\chi$ is below the statistical errors.

The Gell-Mann-Oakes-Renner (GMOR) relation connects the pion mass to the chiral quark mass, $m$, via the pion decay constant $f_{\pi}$ in the chiral limit and the chiral condensate, $-\Sigma$ :

$$
f_{\pi}^{2} m_{\pi}^{2}=2 m \Sigma+\mathcal{O}\left(m^{2}\right)
$$

A direct lattice determination of $f_{\pi}$ from the appropriate current matrix element yields $\sqrt{2} f_{\pi}=$ $Z_{A}\left\langle 0\left|A_{4}\right| \pi\right\rangle / m_{\pi} \rightarrow 0.0496(34) a^{-1}$, in the limit $m_{\pi}^{2} \rightarrow$ 0 [30]. Allowing for an additional systematic error of $10 \%$ on $f_{\pi}$ to account for the fact that we have only determined the axial vector renormalization constants $Z_{A}$ perturbatively, we arrive at the lattice estimate

$$
f_{\pi}=(0.238 \pm 0.030) r_{0}^{-1}=(94 \pm 13) \mathrm{MeV}
$$

at $\kappa=\kappa_{c}$, using the value $r_{0}^{-1} a=0.1476_{-30}^{+47}$ [21]. In combining Eq. (11) with Eq. (11) one expects

$$
\chi=\frac{f_{\pi}^{2} m_{\pi}^{2}}{2 n_{f}}+\mathcal{O}\left(m_{\pi}^{4}\right)
$$

for small pseudoscalar masses and large LeutwylerSmilga parameters, $x=V m \Sigma \gg 1$ [31,32].

In Table IV we display the spatial lattice extents, $L_{\sigma} a m_{\pi}$ as well as $x$ and $\left\langle Q^{2}\right\rangle=\chi V$. We estimate the (scheme independent) combination $m \Sigma$ that appears within $x$ from Eqs. (11) - (12). The topological charge fluctuations $\left\langle Q^{2}\right\rangle$ correspond to the average number of non-interacting instantons that can be accomodated within the simulated lattice volume 33,34. It has

\footnotetext{
${ }^{2}$ Our normalisation conventions are such that $f_{\pi} \approx 92 \mathrm{MeV}$.

${ }^{3}$ This formula as well as the GMOR relation Eq. (11) only apply to $n_{f} \geq 2$ since for $n_{f}=1$ no light pion exists (for a finite number of colours, $\left.N_{c}<\infty\right)$.
} 
TABLE IV. The spatial lattice extents in units of the pion correlation length, the Leutwyler-Smilga parameters, $x=V m \Sigma$ and topological charge fluctuations $\left\langle Q^{2}\right\rangle$.

\begin{tabular}{ccccc}
\hline \hline$\kappa$ & $V / a^{4}$ & $m_{\pi} a L_{\sigma}$ & $x$ & $\left\langle Q^{2}\right\rangle$ \\
\hline 0.1560 & $16^{3} \times 32$ & $7.14(4)$ & $16.0 \pm 4.1$ & $9.1 \pm 0.8$ \\
0.1565 & $16^{3} \times 32$ & $6.39(6)$ & $12.9 \pm 3.2$ & $10.9 \pm 1.7$ \\
0.1570 & $16^{3} \times 32$ & $5.51(4)$ & $9.6 \pm 2.4$ & $7.5 \pm 1.3$ \\
0.1575 & $16^{3} \times 32$ & $4.50(5)$ & $6.4 \pm 1.6$ & $5.4 \pm 0.9$ \\
0.1575 & $24^{3} \times 40$ & $6.65(6)$ & $26.1 \pm 6.6$ & $24.1 \pm 3.9$ \\
0.1580 & $24^{3} \times 40$ & $4.77(7)$ & $13.4 \pm 3.4$ & $17.2 \pm 6.6$ \\
\hline \hline
\end{tabular}

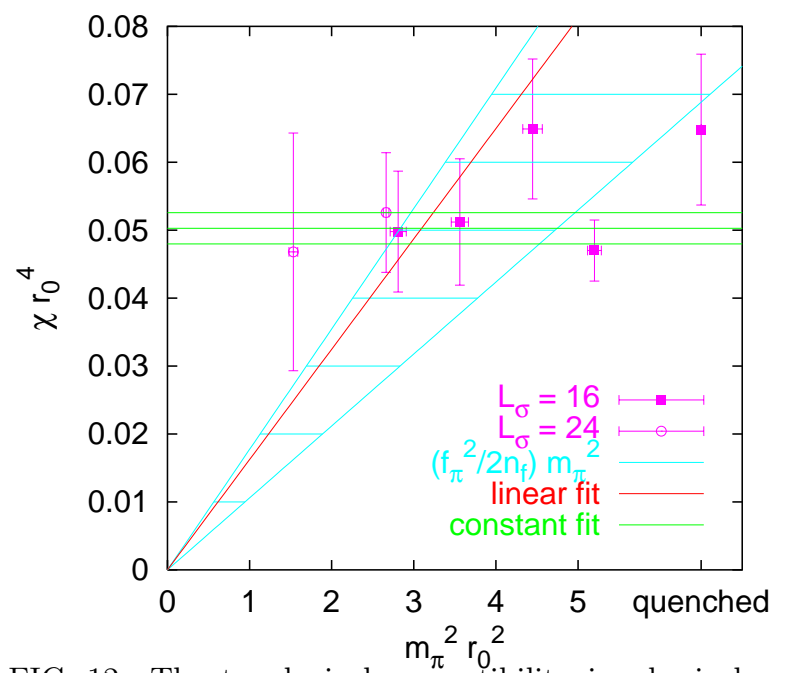

FIG. 12. The topological susceptibility in physical units as a function of $m_{\pi}^{2}$. The right-most data point is from the quenched reference simulation and has not been included into the fits. The dashed error band corresponds to the small mass expectation from the independently determined pion decay constant $f_{\pi}=0.238(30) r_{0}^{-1}$.

been argued [34] that $x>10$ is already a large volume in the Leutwyler-Smilga 31 sense, such that Eq. (13) should safely apply to all but the $16^{3} \times 32$ lattices at $\kappa=0.157$ and $\kappa=0.1575$. Even at the latter, more critical, $\kappa$-value, where we have two lattice volumes that correspond to $x \approx 6$ and $x \approx 25$ at our disposal, we do not find any volume dependence of $\chi$.

In the regime of large $m_{\pi}$ one would expect $\chi$ in units of some reference scale like $r_{0}^{-1}$ to approach the quenched value as a smooth function of $m_{\pi}$. Prior to the comparison with Eq. (13) one should in principle extrapolate the lattice results on $\chi$ to the continuum limit, for instance along a trajectory of constant $m_{\pi} r_{0}$. Having only $\beta=5.6$ data at our disposal we cannot yet perform this extrapolation but conjecture that our results are already sufficiently close to the continuum limit for Eq. (13) to hold. This assumption is plausible in view of the fact that the topological charges obtained from the gluonic and fermionic definitions agree with each other reasonably well (with a renormalization constant $Z_{P}$ of order one) and of our previous studies of light hadronic quan- tities 21, 30.

For the purpose of the present investigation we shall consider the dimensionless topological susceptibility, $\chi r_{0}^{4}$, as a function of the dimensionless combination $m_{\pi}^{2} r_{0}^{2}$ in order to carry out the chiral extrapolation. The results are plotted in Fig. 12, together with the region that is allowed for by the leading order expectation, Eq. (13), for the $f_{\pi}$ value of Eq. (12). All unquenched data except for the point at the heaviest quark mass $\left(m_{\pi}^{2} r_{0}^{2} \approx 5\right)$ are consistent with this expectation. Note that the most chiral data point stems from the run with $\kappa=0.158$ which, as can be seen from Figs. 3 and 11, is not of sufficient statistical quality for the purpose of topological studies. The right-most entry in Fig. 12 is the result of our quenched reference study.

A linear fit, excluding the largest mass point, to the parametrisation (13) renders $f_{\pi}=0.255(11) r_{0}^{-1}$ with $\chi^{2} / N_{D F}=3.93 / 4$. This value compares reasonably well with the expectation, Eq. (12). On the other hand our data are consistent with a mass independence of $\chi$ too: fitting them to a constant yields $\chi r_{0}^{4}=0.0503(23)$ with $\chi^{2} / N_{D F}=2.67 / 5$, in agreement with the quenched reference point, $\chi r_{0}^{4}=0.065(11)$.

Our present study is based on data obtained at a fixed value of $\beta$ and the quark mass is varied by just tuning $\kappa$. It is worthwhile to relate our findings to those recently presented by the UKQCD collaboration [15] who simultaneously vary $\beta$ and $\kappa$ to change the quark mass while keeping the lattice spacing $a$ fixed in units of $r_{0}$. Apart from this they dispose of ensembles of gauge field configuration of comparable statistical sizes, using slightly coarser lattice spacings with an improved fermionic action.

In order to facilitate this comparison we have superimposed both data sets5 in Fig. 13. The data do not clearly contradict each other, however, we are unable to confirm the decrease of the topological susceptibility that has been reported in Ref. [15, at pion masses within the range of present-day lattice simulations.

\section{CONCLUSIONS}

We have demonstrated that Wilson fermions are suitable for lattice studies of topology related effects in the QCD vacuum. We have found agreement between the topological charge computed after cooling from the field strength tensor, $Q=(F, * F) /\left(16 \pi^{2}\right)$, and the fermionic

\footnotetext{
${ }^{4}$ Unfortunately, none of the other collaborations have converted their results into units of $r_{0}$.

${ }^{5}$ The statistical errors quoted by the UKQCD collaboration are somewhat smaller than ours since no signs of autocorrelation effects have been found in their study [35].
} 


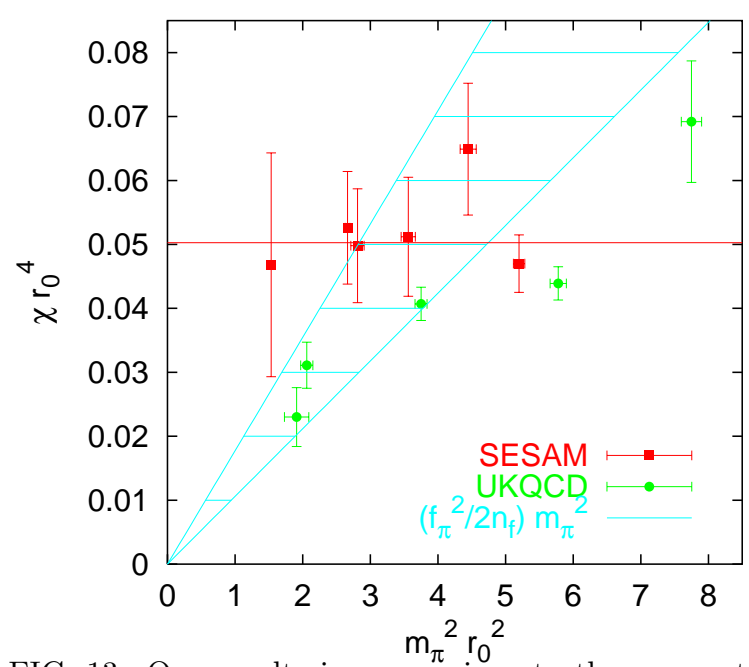

FIG. 13. Our results in comparison to those reported by the UKQCD Collaboration [15. The horizontal line is a constant fit to our data. The dashed error band corresponds to the small mass expectation from the pion decay constant.

definition given by Smit and Vink with a renormalization constant $Z_{P}$ of order one. The trace has been estimated from diagonal improved $Z_{2}$ noisy sources. As the next step we plan to investigate correlations between the topological charge density distribution and eigenvectors of $\gamma_{5} M$.

The topological susceptibility at $\beta=5.6$ has been found to be consistent with that of quenched studies at large sea quark masses. Our data at smaller mass values is consistent with the asymptotic slope in $m_{\pi}^{2}$ that is expected from our independently determined $n_{f}=2$ pion decay constant. However, we are unable to unambiguously confirm the decrease that has recently been reported by the UKQCD collaboration [15] with a different fermionic action. Results obtained by the CP-PACS collaboration [14] who cover a similar range of quark masses on somewhat coarser lattices do not clearly show this tendency either. We plan to clarify this issue in simulations at different $\beta$ values which will enable us to perform a continuum limit extrapolation, and at smaller sea quark masses.

\section{ACKNOWLEDGMENTS}

We thank S. Güsken and P. Überholz for their contributions at an earlier stage of this project. We gratefully acknowledge R. Burkhalter for detecting an important mistake in this article. Our European collaboration was funded by the EU network "Hadron Phenomenology from Lattice QCD" (HPRN-CT-2000-00145). G.B. received support from EU grant HPMF-CT-1999-00353 and PPARC grant PPA/G/O/1998/00559, N.E. from DFG grant Li 701/3-1. B.O. appreciates support from the DFG Graduiertenkolleg "Feldtheoretische und $\mathrm{Nu}$ - merische Methoden in der Statistischen und Elementarteilchenphysik". The HMC productions were run on the APE100 systems at INFN Roma and NIC Zeuthen. We are grateful to our colleagues G. Martinelli and F. Rapuano for the fruitful T $\chi \mathrm{L}$-collaboration. Part of the physics evaluation has been carried out on the ALiCE cluster computer at Wuppertal University.

[1] B. Allés, M. D'Elia, A. Di Giacomo and R. Kirchner, Phys. Rev. D58, 114506 (1998) hep-lat/9711026.

[2] S. Itoh, Y. Iwasaki and T. Yoshié, Phys. Rev. D36, 527 (1987).

[3] R. Narayanan and P. Vranas, Nucl. Phys. B506, 373 (1997) hep-lat/9702005.

[4] M. Teper, Nucl. Phys. Proc. Suppl. 83, 146 (2000) heplat/9909124.

[5] B. Allés, M. D'Elia and A. Di Giacomo, Nucl. Phys. B494, 281 (1997) hep-lat/9605013.

[6] A. Hasenfratz and C. Nieter, Phys. Lett. B439, 366 (1998) hep-lat/9806026.

[7] M. Garcia Perez, O. Philipsen and I. Stamatescu, Nucl. Phys. B551, 293 (1999) hep-lat/9812006.

[8] E. Witten, Nucl. Phys. B156, 269 (1979); G. Veneziano, Nucl. Phys. B159, 213 (1979).

[9] J. Smit and J. C. Vink, Phys. Lett. B194, 433 (1987).

[10] H. Gausterer, J. Potvin, S. Sanielevici and P. Woit, Phys. Lett. B233, 439 (1989).

[11] K. M. Bitar et al. [HEMCGC Collaboration], Phys. Rev. D44, 2090 (1991).

[12] M. Müller-Preußker, in Proc. of the XXVI Int. Conf. on High Energy Physics, Dallas 1992, Ed. J.R. Sanford, AIP Conference Proceedings No. 272, 1545 (1993).

[13] Y. Kuramashi, M. Fukugita, H. Mino, M. Okawa and A. Ukawa, Phys. Lett. B313, 425 (1993).

[14] A. Ali Khan et al. [CP-PACS Collaboration], Nucl. Phys. Proc. Suppl. 83, 162 (2000) hep-lat/9909045.

[15] A. Hart and M. Teper, Nucl. Phys. Proc. Suppl. 83, 476 (2000) hep-lat/9909072; A. Hart [UKQCD Collaboration], hep-ph/0004180; A. Hart and M. Teper [UKQCD Collaboration], hep-lat/0009008.

[16] B. Allés, M. D'Elia and A. Di Giacomo, Phys. Lett. B483, 139 (2000) hep-lat/0004020; B. Allés, M. D'Elia and A. Di Giacomo, Nucl. Phys. Proc. Suppl. 83, 431 (2000) hep-lat/9912012.

[17] A. Hasenfratz, Phys. Lett. B 476, 188 (2000) heplat/9912053].

[18] M. Garcia Perez, Nucl. Phys. Proc. Suppl. 94, 27 (2001) hep-lat/0011026.

[19] B. Allés et al. [T $\chi \mathrm{L}$ Collaboration], Phys. Rev. D58, 071503 (1998) hep-lat/9803008; T. Lippert et al. [SESAM and T $\chi \mathrm{L}$ Collaborations], Nucl. Phys. Proc. Suppl. 73, 521 (1999) hep-lat/9809034; T. Lippert et al. [SESAM Collaboration], Nucl. Phys. Proc. Suppl. 63, 946 (1998) hep-lat/9712020.

[20] B. Orth et al. [SESAM and $\mathrm{T} \chi \mathrm{L}$ Collaborations], in 
preparation.

[21] G. S. Bali et al. [SESAM and T $\chi \mathrm{L}$ Collaborations], Phys. Rev. D62, 054503 (2000) hep-lat/0003012.

[22] T. Lippert et al. [SESAM and T $\chi \mathrm{L}$ Collaborations], in preparation.

[23] P. Di Vecchia, K. Fabricius, G. C. Rossi and G. Veneziano, Nucl. Phys. B192, 392 (1981); P. Di Vecchia, K. Fabricius, G. C. Rossi and G. Veneziano, Phys. Lett. B108, 323 (1982).

[24] B. Berg, Phys. Lett. B104, 475 (1981).

[25] M. Teper, Phys. Lett. B162, 357 (1985).

[26] J. Smit and J. C. Vink, Nucl. Phys. B286, 485 (1987).

[27] J. Viehoff [SESAM Collaboration], Nucl. Phys. Proc. Suppl. 73, 856 (1999) hep-lat/9809073.); J. Viehoff et al. [SESAM Collaboration], Nucl. Phys. Proc. Suppl. 63, 269 (1998) hep-lat/9710050.

[28] T. Struckmann et al. [SESAM and T $\chi \mathrm{L}$ Collaborations], Phys. Rev. D 63, 074503 (2001) hep-lat/0010005.

[29] E. M. Ilgenfritz, B. V. Martemyanov, S. V. Molodtsov, M. Müller-Preußker and Y. A. Simonov, Phys. Rev. D58, 114508 (1998) hep-ph/9712523.

[30] N. Eicker et al. [SESAM collaboration], Phys. Rev. D59, 014509 (1999) hep-lat/9806027.

[31] H. Leutwyler and A. Smilga, Phys. Rev. D 46, 5607 (1992).

[32] S. Dürr, Nucl. Phys. B 594, 420 (2001) heplat/0008022.

[33] T. Schäfer and E. V. Shuryak, Rev. Mod. Phys. 70, 323 (1998) hep-ph/9610451.

[34] S. Dürr, hep-lat/0103011.

[35] A. Hart, private communication. 